\title{
DUNFORD-PETTIS OPERATORS ON BANACH LATTICES ${ }^{1}$
}

BY

\author{
C. D. ALIPRANTIS AND O. BURKINSHAW
}

\begin{abstract}
Consider a Banach lattice $E$ and two positive operators $S, T: E \rightarrow E$ that satisfy $0 \leqslant S \leqslant T$. In $[2,3]$ we examined the case when $T$ is a compact (or weakly compact) operator and studied what effect this had on an operator (such as $S$ ) dominated by $T$. In this paper, we extend these techniques and study similar questions regarding Dunford-Pettis operators. In particular, conditions will be given on the operator $T$, to ensure that $S$ (or some power of $S$ ) is a Dunford-Pettis operator. As a sample, the following is one of the major results dealing with these matters.

THEOREM. Let $E$ be a Banach lattice, and let $S, T: E \rightarrow E$ be two positive operators such that $0 \leqslant S \leqslant T$. If $T$ is compact then

(1) $S^{3}$ is a compact operator (although $S^{2}$ need not be compact);

(2) $S^{2}$ is a Dunford-Pettis and weakly compact operator (although $S$ need not be);

(3) $S$ is a weak Dunford-Pettis operator.

In another direction, our techniques and results will be related to the lattice structure of the Dunford-Pettis operators. For instance, it will be shown that under certain conditions the Dunford-Pettis operators form a band.
\end{abstract}

1. Introduction. It is well known that the class of Dunford-Pettis operators plays an important role in the theory of vector measures and in the theory of Banach spaces in general; for instance, see [7]. This paper will focus on the various interrelationships between compact and Dunford-Pettis operators on Banach lattices. In particular, the lattice structures will be utilized to study what compactness properties are inherited by a positive operator that is dominated by a compact or a Dunford-Pettis operator. In a Banach space, $x_{n} \stackrel{w}{\rightarrow} 0$ will mean that the sequence $\left\{x_{n}\right\}$ converges weakly to zero (i.e., that $\left\langle x^{\prime}, x_{n}\right\rangle=x^{\prime}\left(x_{n}\right) \rightarrow 0$ holds for each continuous linear functional $x^{\prime}$ on the Banach space).

Let $X$ and $Y$ be two Banach spaces. An operator $T: X \rightarrow Y$ is called a Dunford-Pettis operator if $T$ carries weakly convergent sequences onto norm convergent sequences. (Equivalently, if $x_{n} \stackrel{w}{\rightarrow} 0$ implies $\lim \left\|T x_{n}\right\|=0$.) Alternatively: A (bounded linear) operator $T: X \rightarrow Y$ is a Dunford-Pettis operator if and only if $T$ carries relatively weakly compact sets onto norm totally bounded sets.

Observe that a compact operator must be a Dunford-Pettis operator. Clearly, the two notions coincide when $X$ is reflexive. On the other hand, a Dunford-Pettis

Received by the editors April 20, 1981, presented to the Society, January 14, 1982.

1980 Mathematics Subject Classification. Primary 46A40, 46B30, 47B05, 47B55.

Key words and phrases. Banach lattices, positive operators, compact operators, Dunford-Pettis operators.

'Research supported in part by NSF grant MCS 81-00787. 
operator need not be a compact operator, and its adjoint may fail as well to be Dunford-Pettis. For instance, the identity operator $I: l_{1} \rightarrow l_{1}$ is a Dunford-Pettis operator but not weakly compact. Moreover, its adjoint is the identity operator $I: l_{\infty} \rightarrow l_{\infty}$ which is not a Dunford-Pettis operator.

For notation and terminology concerning Banach lattices and locally convex-solid Riesz spaces, not explained below, we follow [11 and 1], respectively. A Banach lattice is said to have an order continuous norm if $x_{\alpha} \downarrow 0$ implies $\left\|x_{\alpha}\right\| \downarrow 0$. Every Banach lattice with order continuous norm is a Dedekind complete Riesz space; see [1, Theorem 10.3, p. 66 or 11, Theorem 5.10, p. 89].

If $E$ is a Banach lattice, then its norm dual $E^{\prime}$ is also a Banach lattice. Besides the norm topology we shall need to consider the absolute weak topologies. The absolute weak topology $|\sigma|\left(E, E^{\prime}\right)$ on $E$ is the locally convex-solid topology of uniform convergence on the order intervals of $E^{\prime}$ and is generated by the family of Riesz seminorms $\left\{\rho_{f}: f \in E^{\prime}\right\}$, where

$$
\rho_{f}(x)=|f|(|x|) \quad \text { for each } x \in E .
$$

The absolute weak topology $|\sigma|\left(E, E^{\prime}\right)$ is consistent with the dual system $\left\langle E, E^{\prime}\right\rangle$, and is a Lebesgue topology if and only if $E$ has order continuous norm. (A locally solid topology $\tau$ on a Riesz space is called a Lebesgue topology if $x_{\alpha} \downarrow 0$ implies $x_{\alpha} \stackrel{\tau}{\rightarrow} 0$.)

Similarly, the absolute weak topology $|\sigma|\left(E^{\prime}, E\right)$ on $E^{\prime}$ is the locally convex-solid topology of uniform convergence on the order intervals of $E$, and is generated by the family of Riesz seminorms $\left\{\rho_{x}: x \in E\right\}$, where

$$
\rho_{x}(f)=|f|(|x|) \quad \text { for each } f \in E^{\prime} .
$$

The topology $|\sigma|\left(E^{\prime}, E\right)$ is always a Lebesgue topology. For details about the absolute weak topologies see [1].

If $T: E \rightarrow F$ is an operator between two Banach lattices, then its adjoint $T^{\prime}: F^{\prime} \rightarrow E^{\prime}$ is the operator defined by

$$
\left\langle T^{\prime} f, x\right\rangle=\langle f, T x\rangle=f(T x)
$$

for each $f \in F^{\prime}$ and $x \in E$. If $T$ is a positive operator (i.e., $x \geqslant 0$ in $E$ implies $T x \geqslant 0$ in $F$ ), then $T^{\prime}$ is likewise positive. A positive operator between Banach lattices is necessarily continuous.

Recall that if $\tau$ is a linear topology on a vector space $E$, then a subset $A$ of $E$ is called $\tau$-totally bounded (or $\tau$-precompact) whenever for each $\tau$-neighborhood $V$ of zero, there exists a finite subset $X$ of $A$ with $A \subseteq X+V$.

LEMMA 1.1. Let $T: E \rightarrow F$ be a positive operator between two Banach lattices, such that $T[0, x]$ is $|\sigma|\left(F, F^{\prime}\right)$-totally bounded for each $0 \leqslant x \in E$. If another operator $S: E \rightarrow F$ satisfies $0 \leqslant S \leqslant T$, then $S[0, x]$ is likewise $|\sigma|\left(F, F^{\prime}\right)$-totally bounded for each $0 \leqslant x \in E$.

Proof. Consider $F$ embedded in $F^{\prime \prime}$, and observe that the Lebesgue topology $|\sigma|\left(F^{\prime \prime}, F^{\prime}\right)$ on $F^{\prime \prime}$ induces $|\sigma|\left(F, F^{\prime}\right)$ on $F$. Thus, $T: E \rightarrow F^{\prime \prime}$ has the property that $T[0, x]$ is $|\sigma|\left(F^{\prime \prime}, F^{\prime}\right)$-totally bounded for each $0 \leqslant x \in E$. From $0 \leqslant S \leqslant T$ and 
[2, Theorem 1.2, p. 291] it follows that $S[0, x]$ is $|\sigma|\left(F^{\prime \prime}, F^{\prime}\right)$-totally bounded for each $0 \leqslant x \in E$. That is, $S[0, x]$ is $|\sigma|\left(F, F^{\prime}\right)$-totally bounded for each $0 \leqslant x \in E$.

The next result describes in terms of disjoint sequences certain weakly compact sets. For the particular case in which we are interested, its proof can be obtained from a classical result of $A$. Grothendieck (this result and similar ones have appeared in $[6,8$, and 10]).

TheOREM 1.2. Let $T: E \rightarrow F$ be an operator between two Banach lattices, and let $0 \leqslant x \in E$. Then $T[0, x]$ is relatively weakly compact if and only if every disjoint sequence $\left\{x_{n}\right\} \subseteq[0, x]$ satisfies $\lim \left\|T x_{n}\right\|=0$.

Proof. Consider the order ideal $A_{x}$ generated by $x$ in $E$. Then $A_{x}$ with the norm $\|y\|_{\infty}=\inf \{\lambda>0:|y| \leqslant \lambda x\}$ is an $A M$-space [11, p. 102]. By Kakutani's representation theorem, $A_{x}$ is Riesz and norm isomorphic to a $C(X)$ space for some Hausdorff compact topological space $X$.

The relative weak compactness of $T[0, x]$ is equivalent to saying that $T: A_{x} \rightarrow F$ is a weakly compact operator, and this in turn is equivalent to $T^{\prime}: F^{\prime} \rightarrow A_{x}^{\prime}$ being weakly compact. On the other hand, if $B^{\prime}$ is the closed unit ball of $F^{\prime}$, then $T^{\prime}\left(B^{\prime}\right)$ is relatively weakly compact if and only if every disjoint sequence of $[0, x]$ converges uniformly to zero on $T^{\prime}\left(B^{\prime}\right)$ (i.e., if and only if $\lim \left\|T x_{n}\right\|=0$ holds for each disjoint sequence $\left\{x_{n}\right\}$ in $[0, x]$ ); see [1, Theorem 10.15, p. 142 or 11, Proposition 9.8, p. 126].

We continue with a useful compactness property of the Dunford-Pettis operators.

LEMMA 1.3. If $E \stackrel{T_{1}}{\rightarrow} F \stackrel{T_{2}}{\rightarrow} G$ are two Dunford-Pettis operators between Banach lattices, then $T_{2} T_{1}[0, x]$ is norm totally bounded for each $0 \leqslant x \in E$.

Proof. If $\left\{x_{n}\right\} \subseteq[0, x]$ is a disjoint sequence, then it is easy to see that $x_{n} \stackrel{w}{\rightarrow} 0$ holds in $E$, and so $\lim \left\|T_{1} x_{n}\right\|=0$. Applying Theorem 1.2 , we see that $T_{1}[0, x]$ is relatively weakly compact, and consequently, $T_{2} T_{1}[0, x]$ must be a norm totally bounded set.

For the next result (and a few others) we shall use the concept of "order-equicontinuity" that was studied extensively in [1]. We shall need the following characterization of the order-equicontinuous sets: If $E$ is a Banach lattice, then a subset $A$ of $E^{\prime}$ is order-equicontinuous on $E$ if and only if for each $0 \leqslant x \in E$ and $\varepsilon>0$ there exists some $g \geqslant 0$ in the ideal generated by $A$ in $E^{\prime}$ such that

$$
\left\langle(|f|-g)^{+}, x\right\rangle \leqslant \varepsilon
$$

holds for all $f \in A$; see [1, Theorem 20.6, p. 135].

It is known that the lattice operations in a Banach lattice are not (in general) weakly sequentially continuous. The next result describes a weak sequential continuity property of the lattice operations, and it can be considered as the heart of this work. (Recall that an operator between two Banach lattices is called regular, if it can be written as a difference of two positive operators.) 
THEOREM 1.4. Let $T: E \rightarrow F$ be a regular operator between two Banach lattices such that $T[0, x]$ is $|\sigma|\left(F, F^{\prime}\right)$-totally bounded for each $0 \leqslant x \in E$. If $x_{n} \stackrel{w}{\rightarrow} 0$ in $E$, then $\left|T x_{n}\right| \stackrel{w}{\rightarrow} 0$ in $F$.

Proof. Assume $x_{n} \stackrel{w}{\rightarrow} 0$ in $E$. Let $0 \leqslant f \in F^{\prime}$ and $\varepsilon>0$. Start by observing that in view of

$$
\left\langle f,\left|T x_{n}\right|\right\rangle=\sup \left\{\left\langle g, T x_{n}\right\rangle: g \in[-f, f]\right\}
$$

(see [1, Theorem 3.5, p. 22]), for each $n$ there exists some $f_{n} \in[-f, f]$ with

$$
\left\langle f,\left|T x_{n}\right|\right\rangle \leqslant 2\left\langle f_{n}, T x_{n}\right\rangle \text {. }
$$

Next note that if we consider $\left\{x_{n}\right\}$ as a sequence of $E^{\prime \prime}$, then $x_{n} \rightarrow 0$ holds in $E^{\prime \prime}$ for $\sigma\left(E^{\prime \prime}, E^{\prime}\right)$, and so, by [1, Theorem 20.20, p. 143] the sequence $\left\{x_{n}\right\}$ is orderequicontinuous on $E^{\prime}$. Thus, there exists some $0 \leqslant x \in E$ with

$$
\left\langle\left|T^{\prime}\right| f,\left(\left|x_{n}\right|-x\right)^{+}\right\rangle \leqslant \varepsilon \text { for each } n \text {. }
$$

Also, from our hypothesis and [2, Theorem 1.3, p. 293] it follows that $T^{\prime}[-f, f]$ is $|\sigma|\left(E^{\prime}, E\right)$-totally bounded. Thus, there exists a finite set $\left\{g_{1}, \ldots, g_{k}\right\} \subseteq T^{\prime}[-f, f]$ such that for each $g \in[-f, f]$ we have

$$
\left\langle\left|T^{\prime} g-g_{i}\right|, x\right\rangle \leqslant \varepsilon \text { for some } 1 \leqslant i \leqslant k .
$$

Since $x_{n} \stackrel{w}{\rightarrow} 0$, there exists some $m$ with $\left|\left\langle g_{i}, x_{n}\right\rangle\right| \leqslant \varepsilon$ for each $1 \leqslant i \leqslant k$ and all $n \geqslant m$.

Now combine (2) and (3) with the inequalities

$$
\begin{aligned}
\left\langle T^{\prime} f_{n}, x_{n}\right\rangle-\left\langle g_{i}, x_{n}\right\rangle & =\left\langle T^{\prime} f_{n}-g_{i}, x_{n}\right\rangle \\
& \leqslant\left\langle\left|T^{\prime} f_{n}-g_{i}\right|,\left(\left|x_{n}\right|-x\right)^{+}\right\rangle+\left\langle\left|T^{\prime} f_{n}-g_{i}\right|, x\right\rangle \\
& \leqslant 2\left\langle\left|T^{\prime}\right| f,\left(\left|x_{n}\right|-x\right)^{+}\right\rangle+\left\langle\left|T^{\prime} f_{n}-g_{i}\right|, x\right\rangle
\end{aligned}
$$

to obtain

$$
\left\langle T^{\prime} f_{n}, x_{n}\right\rangle \leqslant 3 \varepsilon+\left\langle g_{i}, x_{n}\right\rangle \leqslant 4 \varepsilon \text { for all } n \geqslant m .
$$

Finally, in view of (1) it follows from (4) that

$$
\left\langle f,\left|T x_{n}\right|\right\rangle \leqslant 2\left\langle f_{n}, T x_{n}\right\rangle=2\left\langle T^{\prime} f_{n}, x_{n}\right\rangle \leqslant 8 \varepsilon
$$

for all $n \geqslant m$. That is, $\left|T x_{n}\right| \stackrel{w}{\rightarrow} 0$ holds in $F$, and the proof is finished.

Corollary 1.5. Let $E, F$, and $G$ be Banach lattices, let $E \stackrel{S_{1}}{\rightarrow} F \stackrel{S_{2}}{\rightarrow} G$ and $E \stackrel{T_{1}}{\rightarrow} F \stackrel{T_{2}}{\rightarrow} G$ be positive operators with $0 \leqslant S_{i} \leqslant T_{i}$ and let each $T_{i}$ be Dunford-Pettis $(i=1,2)$. If $x_{n} \stackrel{w}{\rightarrow} 0$ holds in $E$, then $\left|S_{2} S_{1} x_{n}\right| \stackrel{w}{\rightarrow} 0$ also holds in $G$.

Proof. By Lemma 1.3, $T_{2} T_{1}[0, x]$ is norm totally bounded (and hence, $|\sigma|\left(G, G^{\prime}\right)$-totally bounded) for each $0 \leqslant x \in E$. But then by Lemma 1.1, $S_{2} S_{1}[0, x]$ is $|\sigma|\left(G, G^{\prime}\right)$-totally bounded for each $0 \leqslant x \in E$, and the conclusion follows from Theorem 1.4. 
2. Compact and Dunford-Pettis operators. Consider a positive operator $S$ that is dominated by a compact operator. In this section we shall investigate when $S$ (or some power of $S$ ) is a Dunford-Pettis operator. But first, a weaker property will be studied.

Let us call an operator $T: X \rightarrow Y$ between two Banach spaces a weak DunfordPettis operator whenever $x_{n} \stackrel{w}{\rightarrow} 0$ in $X$ and $y_{n}^{\prime} \stackrel{w}{\rightarrow} 0$ in $Y^{\prime}$ imply $\lim \left\langle y_{n}^{\prime}, T x_{n}\right\rangle=0$.

In [4] K. T. Andrews calls a bounded subset $A$ of a Banach space $X$ a DunfordPettis set whenever every weakly compact operator with domain $X$ carries $A$ onto a norm totally bounded set. He also characterized these sets as follows: A bounded subset $A$ of a Banach space $X$ is a Dunford-Pettis set if and only if $\left\{x_{n}\right\} \subseteq A$ and $x_{n}^{\prime} \stackrel{w}{\rightarrow} 0$ in $X^{\prime}$ imply $\lim x_{n}^{\prime}\left(x_{n}\right)=0$ [4, Theorem 1, p. 36]. Thus, (by Eberlein's theorem) an operator $T: X \rightarrow Y$ between two Banach spaces is a weak Dunford-Pettis operator if and only if it maps weakly compact subsets of $X$ onto Dunford-Pettis subsets of $Y$. Alternatively: An operator $T: X \rightarrow Y$ between two Banach spaces is a weak Dunford-Pettis operator if and only if for each Banach space $Z$ and each weakly compact operator $S: Y \rightarrow Z$ the operator ST is Dunford-Pettis.

Clearly, a Dunford-Pettis operator is a weak Dunford-Pettis operator. If $X$ and $Y$ are both reflexive, then the notions of "weak Dunford-Pettis" and "Dunford-Pettis" operator coincide. Also, if $Y$ has the Dunford-Pettis property (i.e., $y_{n} \stackrel{w}{\rightarrow} 0$ in $Y$ and $y_{n}^{\prime} \stackrel{w}{\rightarrow} 0$ in $Y^{\prime}$ imply $y_{n}^{\prime}\left(y_{n}\right) \rightarrow 0$ ), then every (bounded linear) operator from $X$ into $Y$ is a weak Dunford-Pettis operator. The weak Dunford-Pettis operators enjoy properties similar to those of compact and Dunford-Pettis operators: They form a closed two sided ideal in the Banach space of all bounded operators from $X$ into $X$. On the other hand, a weak Dunford-Pettis operator need not be a Dunford-Pettis operator; see Example 2.1 below.

If $S$ and $T$ are two positive operators on a Banach lattice, then the compactness of $T$ and the relation $0 \leqslant S \leqslant T$ do not imply (in general) the Dunford-Pettis property (or the weak compactness) of $S$. An example of this type is presented next.

EXAMPLE 2.1. Let $\left\{r_{n}\right\}$ denote the sequence of Rademacher functions on $[0,1]$. That is, $r_{n}(t)=\operatorname{Sgn} \sin \left(2^{n} \pi t\right)$. Consider the operators $S_{1}, T_{1}: L_{1}[0,1] \rightarrow l_{\infty}$ defined by

$$
S_{1}(f)=\left(\int_{0}^{1} f(x) r_{1}^{+}(x) d x, \int_{0}^{1} f(x) r_{2}^{+}(x) d x, \ldots\right)
$$

and

$$
T_{1}(f)=\left(\int_{0}^{1} f(x) d x, \int_{0}^{1} f(x) d x, \ldots\right) .
$$

Then $T_{1}$ is compact (it has rank one), and $0 \leqslant S_{1} \leqslant T_{1}^{\cdot}$ holds. However, $S_{1}$ is not a Dunford-Pettis operator because $r_{n} \stackrel{w}{\rightarrow} 0$ holds in $L_{1}[0,1]$, while

$$
\left\|S_{1} r_{n}\right\|_{\infty} \geqslant \int_{0}^{1} r_{n}(x) r_{n}^{+}(x) d x=\frac{1}{2}
$$

holds for each $n$. Moreover, $S_{1}$ is not weakly compact. (Since $L_{1}[0,1]$ has the Dunford-Pettis property, every weakly compact operator with domain $L_{1}[0,1]$ must 
be a Dunford-Pettis operator.) Also, note that since $l_{\infty}$ has the Dunford-Pettis property, the operator $S_{1}$ is a weak Dunford-Pettis operator.

Now consider the Banach lattice $E=L_{1}[0,1] \oplus l_{\infty}$, and the two operators $S, T: E \rightarrow E$ defined via the matrices

$$
S=\left(\begin{array}{cc}
0 & 0 \\
S_{1} & 0
\end{array}\right) \quad \text { and } \quad T=\left(\begin{array}{cc}
0 & 0 \\
T_{1} & 0
\end{array}\right)
$$

Clearly, $0 \leqslant S \leqslant T$ holds, $T$ is compact, and $S$ is neither a Dunford-Pettis nor a weakly compact operator. However, since $E$ has the Dunford-Pettis property, $S$ is automatically a weak Dunford-Pettis operator.

We continue our discussion with a useful lemma.

Lemma 2.2. Let $E$ and $F$ be two Banach lattices, and let $S, T: E \rightarrow F$ be two positive operators satisfying $0 \leqslant S \leqslant T$ and with $T$ compact. If $A$ is a norm bounded subset of $E^{+}$, then for each $\varepsilon>0$ there exists some $0 \leqslant y \in F$ such that

$$
\left\|(S(x)-y)^{+}\right\|<\varepsilon
$$

holds for all $x \in A$. In particular, $S(A) \subseteq \varepsilon B+[0, y]$, where $B$ is the open unit ball of $F$.

Proof. By the compactness of $T$, there exists a finite subset $\left\{x_{1}, \ldots, x_{n}\right\}$ of $A$ such that for each $x \in A$ we have $\left\|T x-T x_{i}\right\|<\varepsilon$ for at least one $1 \leqslant i \leqslant n$. Now if $y=\sum_{i=1}^{n}\left|T x_{i}\right|$, then the inequalities

$$
(S x-y)^{+} \leqslant(T x-y)^{+} \leqslant\left(T x-T x_{i}\right)^{+} \leqslant\left|T x-T x_{i}\right|
$$

yield

$$
\left\|(S x-y)^{+}\right\|<\varepsilon \quad \text { for all } x \in A .
$$

The last conclusion follows from the identity $S x=(S x-y)^{+}+S x \wedge y$.

We are now in the position to establish that every positive operator, dominated by a compact operator, is a weak Dunford-Pettis operator.

THEOREM 2.3. Let $E$ and $F$ be two Banach lattices, and let $S, T: E \rightarrow F$ be two positive operators satisfying $0 \leqslant S \leqslant T$. If $T$ is compact, then $S$ is a weak Dunford-Pettis operator.

Proof. Let $x_{n} \stackrel{w}{\rightarrow} 0$ in $E$ and $f_{n} \stackrel{w}{\rightarrow} 0$ in $F^{\prime}$. We can assume $\left\|f_{n}\right\| \leqslant 1$ for each $n$. Let $\varepsilon>0$.

Since $\left\{\left|x_{n}\right|\right\}$ is a norm bounded sequence, it follows from Lemma 2.2 that there exists some $0 \leqslant y \in F$ such that

$$
\left\|\left(S\left|x_{n}\right|-y\right)^{+}\right\|<\varepsilon \text { holds for all } n .
$$

Since $\left\{f_{n}\right\}$ as a sequence of $F^{\prime \prime \prime}$ is $\sigma\left(F^{\prime \prime \prime}, F^{\prime \prime}\right)$-convergent to zero, it follows from [1, Theorem 20.20, p. 143] that $\left\{f_{n}\right\}$ is order-equicontinuous on $F^{\prime \prime}$. In particular, by [1, Theorem 20.6, p. 135] this implies the existence of some $0 \leqslant g \in F^{\prime}$ with

$$
\left\langle\left(\left|f_{n}\right|-g\right)^{+}, y\right\rangle \leqslant \varepsilon \text { for all } n \text {. }
$$


Now combine Lemma 1.1 with Theorem 1.4 to see that $\left|S x_{n}\right| \stackrel{w}{\rightarrow} 0$ holds in $F$. Pick some $k$ with $\left\langle g,\left|S x_{n}\right|\right\rangle \leqslant \varepsilon$ for all $n \geqslant k$. But then

$$
\begin{aligned}
\left|\left\langle f_{n}, S x_{n}\right\rangle\right| & \leqslant\left\langle\left|f_{n}\right|,\left|S x_{n}\right|\right\rangle \leqslant\left\langle\left(\left|f_{n}\right|-g\right)^{+},\left|S x_{n}\right|\right\rangle+\left\langle g,\left|S x_{n}\right|\right\rangle \\
& \leqslant\left\langle\left(\left|f_{n}\right|-g\right)^{+}, S\left|x_{n}\right|\right\rangle+\left\langle g,\left|S x_{n}\right|\right\rangle \\
& \leqslant\left\langle\left|f_{n}\right|,\left(S\left|x_{n}\right|-y\right)^{+}\right\rangle+\left\langle\left(\left|f_{n}\right|-g\right)^{+}, y\right\rangle+\left\langle g,\left|S x_{n}\right|\right\rangle \\
& \leqslant \varepsilon+\varepsilon+\varepsilon=3 \varepsilon
\end{aligned}
$$

holds for all $n \geqslant k$. That is, $\lim \left\langle f_{n}, S x_{n}\right\rangle=0$ holds, as required.

REMARKS. (1) If $S$ and $T$ are as in Theorem 2.3, then the preceding proof in actuality shows that: $x_{n} \stackrel{w}{\rightarrow} 0$ in $E$ and $f_{n} \stackrel{w}{\rightarrow} 0$ in $F^{\prime}$ imply $\lim \left\langle\left|f_{n}\right|,\left|S x_{n}\right|\right\rangle=0$.

(2) The preceding proof also shows that if $F$ is $\sigma$-Dedekind complete, then $S$ satisfies the following stronger property: $x_{n} \stackrel{w}{\rightarrow} 0$ in $E$ and $f_{n} \stackrel{w^{*}}{\rightarrow} 0$ in $F^{\prime}$ imply $\lim \left\langle\left|f_{n}\right|,\left|S x_{n}\right|\right\rangle=0$. (Because when $F$ is $\sigma$-Dedekind complete, Theorem 20.20 of [1] can be applied directly to the pair $\left\langle F, F^{\prime}\right\rangle$ instead of $\left\langle F^{\prime \prime}, F^{\prime \prime \prime}\right\rangle$.)

Consider two positive operators $S$ and $T$ on a Banach lattice that satisfy $0 \leqslant S \leqslant T$ and with $T$ compact. The main result in [2] asserts that, in this case, $S^{3}$ must be a compact operator. Although examples in [2] show that $S^{2}$ need not be a compact operator, we shall establish that $S^{2}$ is necessarily a Dunford-Pettis operator.

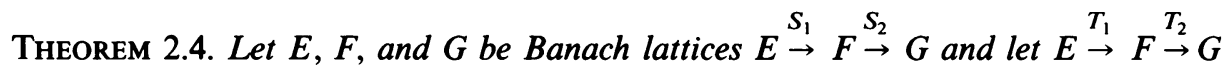
be positive operators with $0 \leqslant S_{i} \leqslant T_{i}(i=1,2)$ and with $T_{1}$ compact. If $T_{2}$ is either Dunford-Pettis or weakly compact, then $S_{2} S_{1}$ is a Dunford-Pettis operator.

Proof. Let $x_{n} \stackrel{w}{\rightarrow} 0$ in $E$. The compactness of $T_{1}$ implies that $T_{1}[0, x]$ is norm totally bounded (and hence, $|\sigma|\left(F, F^{\prime}\right)$-totally bounded) for each $0 \leqslant x \in E$. By Lemma $1.1, S_{1}[0, x]$ is $|\sigma|\left(F, F^{\prime}\right)$-totally bounded for each $0 \leqslant x \in E$. From Theorem 1.4 it follows that

$$
\left|S_{1} x_{n}\right| \stackrel{w}{\rightarrow} 0 \text { in } F .
$$

Assume first that $T_{2}$ is a Dunford-Pettis operator. Then in view of the inequalities

$$
\left|S_{2} S_{1} x_{n}\right| \leqslant S_{2}\left|S_{1} x_{n}\right| \leqslant T_{2}\left|S_{1} x_{n}\right|
$$

we get $\left\|S_{2} S_{1} x_{n}\right\| \leqslant\left\|T_{2}\left|S_{1} x_{n}\right|\right\| \rightarrow 0$, as desired.

Next consider the case when $T_{2}$ is weakly compact. Assume $\lim \left\|S_{2} S_{1} x_{n}\right\| \neq 0$. Then (by passing to a subsequence) we can see that there exists some $\varepsilon>0$ and a sequence $\left\{f_{n}\right\} \subseteq G^{\prime}$ with $\left\|f_{n}\right\|=1$ and $\left|\left\langle f_{n}, S_{2} S_{1} x_{n}\right\rangle\right| \geqslant \varepsilon$ for each $n$. Again, by passing to a subsequence we can assume that $T_{2}^{\prime}\left|f_{n}\right| \stackrel{w}{\rightarrow} f$ in $F^{\prime}$. Hence,

$$
\begin{aligned}
0<\varepsilon & \leqslant\left|\left\langle f_{n}, S_{2} S_{1} x_{n}\right\rangle\right| \leqslant\left\langle\left|S_{2}^{\prime} f_{n}\right|,\left|S_{1} x_{n}\right|\right\rangle \leqslant\left\langle T_{2}^{\prime}\left|f_{n}\right|,\left|S_{1} x_{n}\right|\right\rangle \\
& =\left\langle T_{2}^{\prime}\left|f_{n}\right|-f,\left|S_{1} x_{n}\right|\right\rangle+\left\langle f,\left|S_{1} x_{n}\right|\right\rangle \rightarrow 0,
\end{aligned}
$$

where $\left\langle T_{2}^{\prime}\left|f_{n}\right|-f,\left|S_{1} x_{n}\right|\right\rangle \rightarrow 0$ by the remark after Theorem 2.3, and $\left\langle f,\left|S_{1} x_{n}\right|\right\rangle \rightarrow$ 0 by (*). However, this is impossible, and the proof of the theorem is finished. 
Summarizing the discussion so far, and the main theorems in [ 2 and 3], we can see that the compactness properties of a positive operator dominated by a compact operator increase in strength with the powers of the operator.

TheOREM 2.5. Let $E$ be a Banach lattice, and let $S, T: E \rightarrow E$ be two positive operators satisfying $0 \leqslant S \leqslant T$. If $T$ is compact, then

(1) $S^{3}$ is a compact operator (although $S^{2}$ need not be compact);

(2) $S^{2}$ is Dunford-Pettis and weakly compact (although $S$ need not be);

(3) $S$ is a weak Dunford-Pettis operator.

Proof. Statement (2) follows from Theorem 2.4 and [3, Theorem 4], while (3) follows from Theorem 2.3. Statement (1) is Theorem 2.1 of [2].

If the values of the operators are in a Banach lattice with an order continuous norm, then we can conclude, from $0 \leqslant S \leqslant T$ and the compactness of $T$, that $S$ is a Dunford-Pettis operator.

THEOREM 2.6. Let $E$ and $F$ be two Banach lattices with $F$ having an order continuous norm, and let $S, T: E \rightarrow F$ be two positive operators satisfying $0 \leqslant S \leqslant T$. If $T$ is compact, then $S$ is Dunford-Pettis and weakly compact.

Proof. Assume $x_{n} \stackrel{w}{\rightarrow} 0$ in $E$, and let $\varepsilon>0$. Combining Lemma 1.1 with Theorem 1.4, we see that $\left|S x_{n}\right| \stackrel{w}{\rightarrow} 0$ holds in $F$, i.e., $\left|S x_{n}\right| \rightarrow 0$ holds for $|\sigma|\left(F, F^{\prime}\right)$.

Since $\left\{\left|x_{n}\right|\right\}$ is a norm bounded sequence, there exists (by Lemma 2.2) some $0 \leqslant y \in F$ such that

$$
\left\|\left(S\left|x_{n}\right|-y\right)^{+}\right\|<\varepsilon \text { holds for all } n .
$$

The inequality $\left|S x_{n}\right| \wedge y \leqslant\left|S x_{n}\right|$ shows that $\left|S x_{n}\right| \wedge y \rightarrow 0$ holds for $|\sigma|\left(F, F^{\prime}\right)$. But then the order continuity of the norm of $F$ coupled with $0 \leqslant\left|S x_{n}\right| \wedge y \leqslant y$ implies $\left\|\left|S x_{n}\right| \wedge y\right\| \rightarrow 0$; see [2, Theorem 1.4, p. 293]. Choose some $k$ with $\left\|\left|S x_{n}\right| \wedge y\right\|<\varepsilon$ for all $n>k$.

Now from the inequality

$$
\left|S x_{n}\right|=\left(\left|S x_{n}\right|-y\right)^{+}+\left|S x_{n}\right| \wedge y \leqslant\left(S\left|x_{n}\right|-y\right)^{+}+\left|S x_{n}\right| \wedge y
$$

it follows that

$$
\left\|S x_{n}\right\| \leqslant\left\|\left(S\left|x_{n}\right|-y\right)^{+}\right\|+\left\|\left|S x_{n}\right| \wedge y\right\| \leqslant \varepsilon+\varepsilon=2 \varepsilon
$$

for all $n>k$. That is, $\lim \left\|S x_{n}\right\|=0$, so that $S$ is a Dunford-Pettis operator. The weak compactness of $S$ follows from [3, Theorem 7].

The hypotheses of the preceding theorem are not (in general) enough to insure that $S$ is a compact operator; see for instance [2, Example 3.1, p. 297].

Consider a Banach lattice $E$ with order continuous norm and two positive operators $S, T: E \rightarrow E$ that satisfy $0 \leqslant S \leqslant T$. If $T$ is compact, then we know by now that $S^{2}$ is compact [2, Theorem 2.2, p. 295], $S$ is Dunford-Pettis (Theorem 2.6), and that $S$ need not be compact [2, Example 3.2, p. 297]. Also, a Dunford-Pettis operator is not necessarily a compact operator. Surprisingly enough, we shall show next that if both $E$ and $E^{\prime}$ have order continuous norms, then every positive operator dominated by a positive Dunford-Pettis operator must be a compact operator. (If $E$ and $E^{\prime}$ have order continuous norms, then $E$ need not be reflexive; for instance, let $E=c_{0}$.) 
THEOREM 2.7. Let $E$ be a Banach lattice such that both $E$ and $E^{\prime}$ have order continuous norms, and let $T: E \rightarrow E$ be a positive Dunford-Pettis operator. Then $T$ is a compact operator (and hence, in this case if $S: E \rightarrow E$ satisfies $0 \leqslant S \leqslant T$, then $S$ is a compact operator $)$.

Proof. Let $0 \leqslant x \in E$. Since $E$ has order continuous norm the order interval $[0, x]$ is weakly compact [11, Theorem 5.10, p. 89], and hence, by the Dunford-Pettis property of $T$ the set $T[0, x]$ is norm compact.

Now let $\varepsilon>0$. Denote by $B$ the open unit ball of $E$, and $B^{+}=\{x \in B: x \geqslant 0\}$. Our objective is to show that there exists some $0 \leqslant y \in E$ such that

$$
T\left(B^{+}\right) \subseteq \varepsilon B+T[0, y] .
$$

If this is done, then since we already know that $T[0, y]$ is a norm compact set, it will follow from (**) that $T\left(B^{+}\right)$is a norm totally bounded set (i.e., that $T$ is a compact operator).

Observe first that every disjoint sequence $\left\{x_{n}\right\}$ of $B^{+}$satisfies $x_{n} \stackrel{w}{\rightarrow} 0$. Indeed, if $0 \leqslant f \in E^{\prime}$, then write $f=h_{n}+g_{n}$, where $0 \leqslant h_{n} \in N_{n}=\left\{h \in E^{\prime}:|h|\left(x_{n}\right)=0\right\}$ and $0 \leqslant g_{n} \in C_{n}=N_{n}^{d}$. The disjointness of $\left\{x_{n}\right\}$ implies the disjointness of $\left\{g_{n}\right\}$ [1, Theorem 3.10, p. 24], and in view of $0 \leqslant g_{n} \leqslant f$, the order continuity of the norm in $E^{\prime}$ implies $\lim \left\|g_{n}\right\|=0$. Therefore, the inequalities $0 \leqslant f\left(x_{n}\right)=g_{n}\left(x_{n}\right) \leqslant\left\|g_{n}\right\|$ imply $\lim f\left(x_{n}\right)=0$.

Next note that if $\left\{x_{n}\right\}$ is a disjoint sequence of $B$, then by the above $\left|x_{n}\right| \stackrel{w}{\rightarrow} 0$ in $E$, and so, $\lim \left\|T x_{n}\right\|=0$. In other words, every disjoint sequence of $B^{+}$converges uniformly to zero on the solid hull of $T^{\prime}\left(B^{\prime}\right)$, where $B^{\prime}$ is the open unit ball of $E^{\prime}$. Now observe that statement (ii) of [1, Theorem 21.7, p. 151] (applied to the pair $B$ and the solid hull of $T^{\prime}\left(B^{\prime}\right)$ ) holds. But then statement (iii) of the same theorem shows that there exists some $0 \leqslant y \in E$ satisfying $\left\|T(|x|-y)^{+}\right\|<\varepsilon$ for all $x \in B$. Now if $x \in B^{+}$, then $x=(x-y)^{+}+x \wedge y$ implies $T(x)=T(x-y)^{+}+T(x \wedge y)$ $\in \varepsilon B+T[0, y]$. That is, (**) holds, and so, $T$ is a compact operator.

The parenthetical part follows immediately from the main theorem of [9]; see also [2, Theorem 2.3].

The referee has remarked that the proof of Theorem 2.7 shows that in actuality the following theorem holds.

THEOREM 2.8. Let $E$ and $F$ be Banach lattices such that $E$ and $E^{\prime}$ have order continuous norms, and let $T: E \rightarrow F$ be a positive Dunford-Pettis operator. Then $T$ is a compact operator (and hence, if $S: E \rightarrow F$ satisfies $0 \leqslant S \leqslant T$, then $S$ is weakly compact and weak Dunford-Pettis).

For the proof of the parenthetical part of use [3, Theorem 7] and Theorem 2.3.

3. Dunford-Pettis operators. In this section we shall investigate positive operators that are dominated by Dunford-Pettis operators. Conditions will be given to ensure that various powers of an operator are Dunford-Pettis. The first result is the "Dunford-Pettis version" of the main theorem in [2]. 
THeOREM 3.1. Let $E$ be a Banach lattice, and let $S, T: E \rightarrow E$ be positive operators satisfying $0 \leqslant S \leqslant T$. If $T$ is Dunford-Pettis, then $S^{3}$ is likewise a Dunford-Pettis operator.

Proof. Let $x_{n} \stackrel{w}{\rightarrow} 0$ in $E$. Then by Corollary $1.5,\left|S^{2} x_{n}\right| \stackrel{w}{\rightarrow} 0$ also holds. Thus, in view of the inequalities

$$
\left|S^{3} x_{n}\right| \leqslant S\left|S^{2} x_{n}\right| \leqslant T\left|S^{2} x_{n}\right|,
$$

we get $\left\|S^{3} x_{n}\right\| \leqslant T\left|S^{2} x_{n}\right| \| \rightarrow 0$, as desired.

The arguments of the proof of the preceding theorem show that in actuality the following general version holds. An alternate proof based on Theorem 3.1 will be presented below.

THEOREM 3.2. Let $E, F, G$, and $H$ be Banach lattices, and let $E \stackrel{S_{1}}{\rightarrow} \underset{\rightarrow}{\rightarrow} \stackrel{S_{2}}{\rightarrow} G \stackrel{S_{3}}{\rightarrow} H$ and $E \stackrel{T_{1}}{\rightarrow} F \stackrel{T_{2}}{\rightarrow} G \stackrel{T_{3}}{\rightarrow} H$ be positive operators such that $0 \leqslant S_{i} \leqslant T_{i}(i=1,2,3)$ holds. If each $T_{i}$ is a Dunford-Pettis operator, then $S_{3} S_{2} S_{1}$ is likewise a Dunford-Pettis operator.

Proof. Consider the Banach lattice $L=E \oplus F \oplus G \oplus H$, and then define the operators $S, T: L \rightarrow L$ via the matrices.

$$
S=\left(\begin{array}{cccc}
0 & 0 & 0 & 0 \\
S_{1} & 0 & 0 & 0 \\
0 & S_{2} & 0 & 0 \\
0 & 0 & S_{3} & 0
\end{array}\right) \text { and } T=\left(\begin{array}{cccc}
0 & 0 & 0 & 0 \\
T_{1} & 0 & 0 & 0 \\
0 & T_{2} & 0 & 0 \\
0 & 0 & T_{3} & 0
\end{array}\right)
$$

Clearly, $T$ is a Dunford-Pettis operator, and $0 \leqslant S \leqslant T$ holds. By Theorem 3.1,

$$
S^{3}=\left(\begin{array}{cccc}
0 & 0 & 0 & 0 \\
0 & 0 & 0 & 0 \\
0 & 0 & 0 & 0 \\
S_{3} S_{2} S_{1} & 0 & 0 & 0
\end{array}\right)
$$

must be a Dunford-Pettis operator, and therefore, $S_{3} S_{2} S_{1}$ is likewise a Dunford-Pettis operator.

The next result is the Dunford-Pettis analogue of Theorem 2.5 of [2]. Note the contrast in the hypotheses between the two theorems; here what is important is the domain, while in [2, Theorem 2.5] it was the range.

THeOrem 3.3. Let $E, F$, and $G$ be Banach lattices, let $E \stackrel{S_{1}}{\rightarrow} F \stackrel{S_{2}}{\rightarrow} G$ and $E \stackrel{T_{1}}{\rightarrow} F \stackrel{T_{2}}{\rightarrow} G$ be positive operators with $0 \leqslant S_{i} \leqslant T_{i}$ and let each $T_{i}$ be Dunford-Pettis $(i=1,2)$. If $E$ has an order continuous norm, then $S_{2} S_{1}$ is a Dunford-Pettis operator.

Proof. By the order continuity of the norm in $E$ each order interval of $E$ is weakly compact, and so, $T_{1}[0, x]$ is norm compact for each $0 \leqslant x \in E$. By Lemma $1.1, S_{1}[0, x]$ is $|\sigma|\left(F, F^{\prime}\right)$-totally bounded for each $0 \leqslant x \in E$.

Now assume $x_{n} \stackrel{w}{\rightarrow} 0$ in $E$. By Theorem $1.4,\left|S_{1} x_{n}\right| \stackrel{w}{\rightarrow} 0$ holds in $F$, and so, $\left\|T_{2}\left|S_{1} x_{n}\right|\right\| \rightarrow 0$. The desired conclusion now follows from the inequalities

$$
\left|S_{2} S_{1} x_{n}\right| \leqslant S_{2}\left|S_{1} x_{n}\right| \leqslant T_{2}\left|S_{1} x_{n}\right| \text {. }
$$


Now operators on $A M$ and $A L$ spaces will be considered. The next result shows that if the domain is an $A M$-space, then the condition $0 \leqslant S \leqslant T$ and the fact that $T$ is a Dunford-Pettis operator imply that $S$ is also Dunford-Pettis.

THEOREM 3.4. Let $E$ be a AM-space, let $F$ be a Banach lattice, and let $S, T: E \rightarrow F$ be two positive operators satisfying $0 \leqslant S \leqslant T$. If $T$ is Dunford-Pettis, then $S$ is likewise Dunford-Pettis.

Proof. Let $x_{n} \stackrel{w}{\rightarrow} 0$ in $E$. Since $E$ is an $A M$-space, its lattice operations are weakly sequentially continuous [11, Proposition 7.6, p. 106], and so, $\left|x_{n}\right| \stackrel{w}{\rightarrow} 0$ also holds in $E$. Therefore, $\left\|T\left|x_{n}\right|\right\| \rightarrow 0$, and our conclusion follows from the inequalities $\left|S x_{n}\right| \leqslant S\left|x_{n}\right| \leqslant T\left|x_{n}\right|$.

For regular operators, the next result gives a simple characterization of the Dunford-Pettis operators from a Banach lattice with an order continuous norm into an $A L$-space.

THEOREM 3.5. Let $E$ be a Banach lattice with an order continuous norm, and let $F$ be an $A L$-space. Then for a regular operator $T: E \rightarrow F$ the following statements are equivalent:

(1) $T$ is a Dunford-Pettis operator.

(2) $T$ maps order intervals onto norm compact sets.

Proof. (1) $\Rightarrow(2)$ Note first that the order continuity of the norm in $E$ is equivalent to the weak compactness of its order intervals. Since $T$ is a Dunford-Pettis operator, it will map an order interval of $E$ onto a norm compact subset of $F$.

$(2) \Rightarrow(1)$ Let $x_{n} \stackrel{w}{\rightarrow} 0$ in $E$. Then by Theorem $1.4,\left|T x_{n}\right| \stackrel{w}{\rightarrow} 0$ holds in $F$. If $e(x)=\left\|x^{+}\right\|-\left\|x^{-}\right\|$for each $x \in F$, then $e \in F^{\prime}$ and $\|x\|=e(|x|)$ holds. Thus, $\left\|T x_{n}\right\|=e\left(\left|T x_{n}\right|\right) \rightarrow 0$, so that $T$ is a Dunford-Pettis operator.

Recall that if $E$ and $F$ are two Riesz spaces with $F$ Dedekind complete, then the vector space $\varrho_{b}(E, F)$ of all regular operators from $E$ into $F$ forms a Dedekind complete Riesz space. Applying [2, Theorem 1.2, p. 291] the following result is now immediate from the preceding theorem.

COROLlaRY 3.6. Let $E$ be a Banach lattice with an order continuous norm, and let $F$ be an AL-space. Then the collection of all Dunford-Pettis operators of $\varrho_{b}(E, F)$ forms $a$ band in $L_{b}(E, F)$.

If $E$ and $F$ are both $A L$-spaces, then the bounded operators from $E$ into $F$ are precisely the regular operators from $E$ into $F$ [11, Theorem 1.5, p. 232]. Thus, for two $A L$-spaces Corollary 3.6 takes the following form.

Corollary 3.7. If $E$ and $F$ are two AL-spaces, then the collection of all DunfordPettis operators from $E$ into $F$ forms a band of $L(E, F)$.

The preceding corollary was first proved in [9, Corollary 6.2, p. 313]. Also, it was established in [5, p. 38] that the Dunford-Pettis operators in $\mathcal{E}\left(L_{1}, L_{1}\right)$, where $L_{1}=L_{1}[0,1]$, form a sublattice. 
Open problem. The following interesting theorem was obtained in [9].

TheOREM (DodDS-FremLIN). Let $E$ and $F$ be Banach lattices with $E^{\prime}$ and $F$ having order continuous norms. If $S, T: E \rightarrow F$ satisfy $0 \leqslant S \leqslant T$ and $T$ is a compact operator, then $S$ is likewise a compact operator.

An alternative proof of this result was presented in [2].

Problem. Is the analogue of the above theorem true for Dunford-Pettis operators? That is, if $E$ and $F$ are as in the preceding theorem and $S, T: E \rightarrow F$ satisfy $0 \leqslant S \leqslant T$ with $T$ Dunford-Pettis, is then $S$ a Dunford-Pettis operator?

Note that if $E, E^{\prime}$, and $F$ all have order continuous norms, then the proof of Theorem 2.7 shows that the answer is affirmative (in fact, $S$ and $T$ are both compact).

\section{REFERENCES}

1. C. D. Aliprantis and O. Burkinshaw, Locally solid Riesz spaces, Academic Press, New York and London, 1978.

2. __ Positive compact operators on Banach lattices, Math. Z. 174 (1980), 289-298.

3. On weakly compact operators on Banach lattices, Proc. Amer. Math. Soc. 83 (1981), $573-578$.

4. K. T. Andrews, Dunford-Pettis sets in the space of Bochner integrable functions, Math. Ann. 241 (1979), 35-41.

5. J. Bourgain, Dunford-Pettis operators on $L^{1}$ and the Radon-Nikodym property, Israel J. Math. 37 (1980), 34-47.

6. O. Burkinshaw, Weak compactness in the order dual of a vector lattice, $\mathrm{Ph}$. D. thesis, Purdue University, 1972.

7. J. J. Diestel and J. J. Uhl, Jr., Vector measures, Math. Surveys, No. 15, Amer. Math. Soc., Providence, R. I., 1977.

8. P. G. Dodds o-weakly compact mappings on Riesz spaces, Trans. Amer. Math. Soc. 214 (1975), 389-402.

9. P. G. Dodds and D. H. Fremlin, Compact operators in Banach lattices, Israel J. Math. 34 (1979), 287-320.

10. M. Duhoux, o-weakly compact mappings from a Riesz space to a locally convex space, Bull. Math. Soc. Sci. Math. R. S. Roumanie (N. S.) 22 (1978), 371-378.

11. H. H. Schaefer, Banach lattices and positive operators, Springer-Verlag, Berlin and New York, 1974.

DEPARTMENT OF MATHEMATICAL SCIENCES, INDIANA UNIVERSITY-PURdUE UNIVERSITY AT INDIANAPOLIS, INDIANAPOLIS, INDIANA 46205 